\section{Long-acting Thyroid Stimulator in Thyrotoxic Monozygous Twins}

British Medical fournal, 1970, 3, 205

Thyrotoxicosis in identical twins has been noted previouslyHassan et al. (1966) described four such pairs-but it was regarded as extremely rare, at least up to 1961 (Lowenstein, 1961). Since then a number of cases have been reported, including also other disorders of the thyroid, such as Hashimoto's thyroiditis, and one in which there was a different disorder in each twin-thyrotoxicosis in the one and lymphadenoid goitre in the other (Jayson et al., 1967). These workers further carried out an assay for long-acting thyroid stimulator (L.A.T.S.) in the sera of their twin patients and found it in both. While L.A.T.S. is detectable in up to $80 \%$ of thyrotoxic sera, and might therefore be expected to be present in any two cases chosen at random, it is believed that this is the first reported case in which L.A.T.S. has been assayed in monozygous twins suffering from thyrotoxicosis.

\section{Case Reports}

The twins were born in March 1947 of healthy parents. There was a family history of thyroid disorder on both sides.

Twin 1 presented in 1964 aged 17 with recent rapid loss of weight $(14 \mathrm{lb} ; 6.4 \mathrm{~kg}$.), ocular prominence, restlessness, and irritability. She had hot moist hands, a moderate fine tremor, pronounced bilateral true exophthalmos, with oedema of the eyelids, and a heart rate of 120. There was moderate smooth uniform enlargement of the thyroid gland, with an overlying bruit and thrill. As this was a clinically typical case of primary Graves' disease, she was treated with carbimazole for two years. There was an excellent response and after five years there was no evidence of relapse, but eye signs were still apparent. The thyroid gland was no longer palpable.

Twin 2 presented in 1968 aged 21 with a two-month history of nervousness, lassitude, sweating, and fullness of the neck, but no significant loss of weight. There was pronounced lid retraction and lid-lag, but no true exophthalmos, fine tremor of the outstretched hands, and a heart rate of 110 . The thyroid gland was moderately enlarged, smooth, and soft, with an overlying bruit. Serum P.B.I. was $19 \mu \mathrm{g} . / 100 \mathrm{ml}$. She was treated with carbimazole, with early obvious response, and was clinically euthyroid within three months. She remained fully controlled after 14 months on continued treatment (see Table).

Description of Twins and Evidence for Monozygosity.-They were normally developed healthy girls. Their hair colour and texture, shape of head, position and shape of ears, hand structure, and colour of iris were the same. Both were of above average intelligence and had taken university degrees. They were concordant on the basis of serum isoenzyme tests and also on the blood-group phenotypes. The parents were also tested, and with this additional information it can be calculated that the probability of the twins being monozygotic is 0.99 .

\section{COMMENT}

The genetic factor in thyroid disorders is well recognized (Bartels, 1941), but more recently the evidence has pointed to a recessive factor, favouring the development of primary thyrotoxicosis, but not of secondary toxic nodular goitre (Martin and Fisher, 1945, 1951). This was five years before the discovery of L.A.T.S. in 1956, which now appears to provide an explanation of the mechanism and, according to Hassan et al. (1966), there may well be an inherited immunological instability, resulting in the development of antibodies interacting with thyroid cell (and gastric parietal cell) antigen in some cases. In fact, toxic diffuse goitre appears to be "a genetically determined autoimmune disorder." Jayson et al. (1967) described cases further pointing to this conclusion and quoted the findings of Harvald and Hauge (1956) that there is "an increased concordance rate of the disease in monozygotic as opposed to dizygatic pairs." Antinuclear factor was present at weak titres in the present cases; it is more commonly found in children than in adults (Doniach et al., 1965).

In this example of monozygotic twins with diffuse goitre L.A.T.S. has been shown to be present. This assay has not as yet been done on the parents, who, incidentally, were negative for thyroid and gastric autoantibodies and for antinuclear factors.

I am indebted to Dr. A. H. Cameron of the Children's Hospital, Birmingham, and Mrs. J. Wingham of the Birmingham Blood Transfusion Service for the blood grouping; to Dr. Elizabeth Robson of the Galton Laboratory, University College, London, for the serum enzyme tests and their interpretation; to Dr. Deborah Doniach, Department of Immunology, Middlesex Hospital, for the autoantibody tests and advice; and to Dr. D. J. El Kabir, Department of Human Anatomy, Oxford University, for the L.A.T.S. assays.

W. M. PRIEST, M.D., F.R.C.P.

Warneford General Hospital, Leamington Spa, Warwickshire. REFERENCES

Bartels, E. D. (1941). Heredity in Graves' Disease. Munksgaard, Copenhagen. Doniach, D., Nilsson, L.
Scandinavica, 54, 260 .

Harvald, B., and Hauge, M. (1956). Danish Medical Bulletin, 3, 150.

Hassan, T. H. A., Greig, W. R., Boyle, J. A., Boyle, I. T., and Wallace, T. J. (1966). Lancet, 2, 306

Jayson, M. I. V., Doniach, D., Benhamou-Glynn, N., Roitt, I. M., and El Kabir, D. J. (1967). Lancet, $2,15$.

Lowenstein, J. M. (1961). Fournal of the American Medical Association, 176, 377 .

Martin. L., and Fisher, R. A. (1945). Quarterly fournal of Medicine, 14, 207.

Martin, L., and Fisher, R. A. (1951). Quarterly fournal of Medicine, 20, 293. 